\title{
AN EXPLORATORY STUDY ON SECONDARY EDUCATION STUDENTS' PREFERENCES REGARDING SCIENCE TEACHING METHODS: THE CASE OF A HIGH SCHOOL
}

\author{
SOLAZ-PORTOLÉS, Joan Josep ${ }^{1 *}$; DEL CAMPO, Óscar J.; SANJOSÉ, Vicent ${ }^{3}$; \\ 1,2,3 Universitat de València, Facultat de Magisteri, Departament de Didàctica de les Ciències Experimentals $\mathrm{i}$ \\ Socials, Avinguda dels Tarongers 4, 46022 Valencia (Spain) \\ ${ }^{*}$ Autor correspondente \\ e-mail: Joan.Solaz@uv.es
}

Received 08 June 2016; accepted 12 June 2016

\section{RESUMEN}

En este estudio se pretende llevar a cabo una primera aproximación a las preferencias de metodología en la enseñanza de las ciencias de los estudiantes de educación secundaria. También se analiza la influencia del nivel académico y género sobre dichas preferencias. Han participado 240 estudiantes de educación secundaria españoles (112 mujeres y 128 hombres) de diferentes niveles académicos (pero todos pertenecen al mismo centro educativo), a los que se ha administrado un cuestionario de opción múltiple compuesto por 6 ítems. Cada ítem tiene cuatro opciones en las que se ofrece un enfoque de enseñanza. El análisis estadístico de los resultados obtenidos nos permite concluir que: a) Los estudiantes tienen predilección por enfoques instruccionales centrados en el estudiante; b) El nivel académico influye significativamente sobre las preferencias en la metodología de enseñanza, pero el género no.

Palabras clave: Enseñanza de las ciencias, educación media, metodología de la enseñanza, nivel educativo, género.

\begin{abstract}
This study aims to carry out an initial approach to the secondary education students' preferences regarding science teaching methods. The study also analyses the influence of academic level and gender on these preferences. Two hundred and forty spanish secondary education students (112 women and 128 men) at different educational levels (but they are all from the same high school) have participated in this research. All of them have been administered a multiple choice test of 6 items each with four options. In these items each option offers a way of teaching. The statistical analysis of the results obtained allow us to conclude that: a) Students show a significant predilection for student-centered teaching methods; and b) Academic level has a significant effect on teaching method preferences among students, but gender does not.
\end{abstract}

Keywords: Science education, secondary education, teaching methodology, academic level, gender.

PERIÓDICO TCHÊ QUÍMICA • www.periodico.tchequimica.com • Vol. 13 N. 26

- ISSN 1806-0374 (impresso) • ISSN 1806-9827 (CD-ROM) • ISSN 2179-0302 (meio eletrônico)

(C) 2016. Porto Alegre, RS. Brasil 


\section{INTRODUCCIÓN}

La forma en que un estudiante consigue, procesa, retiene y recupera la información se ha denominado estilo de aprendizaje (Felder y Henriques, 1995). Dunn y Dunn (1993) formularon un modelo para definir los distintos estilos de aprendizaje que incorpora hasta veintidós elementos en cinco grandes grupos: ambiente, afectividad, preferencias sociológicas, preferencias fisiológicas y preferencias de procesamiento cognitivo. Tras muchos años de investigación, Dunn y Griggs (2007) pusieron a prueba diferentes estrategias instruccionales para demostrar su efectividad en función de los estilos de aprendizaje que mostraban los estudiantes. De hecho, en el ámbito de las didáctica de las ciencias, ya había firmes defensores del emparejamiento entre estrategias instruccionales y estilos de aprendizaje de los estudiantes (Kempa, 1995). Sin embargo, tras una revisión y análisis de la literatura, Pashler et al. (2008) concluyeron que la hipótesis de que la utilización de una metodología de enseñanza acorde con las preferencias mostradas por los estudiantes en su estilo de aprendizaje mejora el rendimiento en el aprendizaje, no tiene suficientes evidencias empíricas como para ser validada.

En este sentido, citaremos aquí dos trabajos muy representativos de resultados de investigaciones que pueden resultar contradictorios en esta cuestión del emparejamiento instrucción/estilo de aprendizaje. Así, en un experimento muy concreto (donde la variable estilo de aprendizaje tiene dos valores visual o auditivo) se ha puesto en evidencia que la utilización de una metodología de enseñanza en consonancia con el estilo de aprendizaje no genera diferencias estadísticamente significativas en el aprendizaje (Rogowsky et al., 2015). En cambio, en la investigación llevada a cabo por Garcia-Ros et al. (2008) se han encontrado correlaciones positivas y bajas, pero estadísticamente significativas, entre las preferencias instruccionales de estudiantes universitarios de primer curso, sus estilos de aprendizaje y motivación para el aprendizaje.

Desde hace algún tiempo se tiende a hacer pivotar los modelos de enseñanza sobre dos ejes: el estudiante y el profesor. Así, se habla de metodologías centradas en el aprendiz y de metodologías centradas en el profesor, y de cómo transformar las últimas en las primeras para mejorar los resultados del aprendizaje (Brown, 2003; Catalano y Catalano, 1999). De las metodologías de enseñanza centradas en el profesor, la máxima representante es la metodología tradicional de enseñanza, que parece la más representativa entre los profesores en activo, y que se basa en la transmisión verbal de contenidos disciplinares (Porlán-Ariza et al., 1998).

Según Weimer (2013), algunas de las principales características de un enfoque de enseñanza centrado en el estudiante son: se desarrollan estrategias de aprendizaje y autoconciencia en el estudiante; la tarea del profesor se focaliza en el aprendizaje del estudiante y en crear ambientes de aprendizaje que resulten de interés y fructíferos para el estudiante; la evaluación constituye una parte muy importante del aprendizaje; y se promueve la colaboración entre estudiantes. Muchos investigadores han desarrollado y utilizado modelos instruccionales que encajan en los criterios anteriormente mencionados de una metodología centrada en el estudiante. Algunas denominaciones que se han dado a estos modelos son: aprendizaje activo (Bonwell y Eison, 1991), aprendizaje colaborativo (Bruffee, 1999), aprendizaje basado en la indagación (Marx et al., 2004), aprendizaje cooperativo (Johnson et al., 1998) o enseñanza mediante investigación dirigida (Gil, 1993).

En el magnífico trabajo de revisión de la literatura de Michael (2006) se ponen de manifiesto las mejoras significativas en los resultados de aprendizaje cuando se utilizan estrategias de enseñanza centradas en el estudiante. Estudios posteriores han vuelto a corroborar estos resultados (Anjur, 2011; Armbruster et al., 2009). Especial mención debe hacerse del amplio estudio de Granger et al. (2012), que confirmó nuevamente la bondad del enfoque instruccional centrado en el estudiante y que se publicó en una revista de tanta importancia e impacto como Science.

El género, la edad y la motivación son otras variables que pueden tener cierta relevancia en los procesos de enseñanza/aprendizaje de las ciencias en la escuela secundaria. Slater et al. (2007) han mostrado que no existen diferencias significativas entre hombres y mujeres en las preferencias de estilos de aprendizaje, y Weber y Custer (2005) han hallado que las preferencias 
en los enfoques instruccionales son muy similares en ambos sexos. La variable edad, sin embargo, se revela decisiva puesto que las creencias epistemológicas y las formas de encarar el aprendizaje cambian bastante con la edad e influyen en el rendimiento académico (Cano, 2005). Íntimamente ligada con la variable edad en la educación secundaria está el nivel académico, y Chen (2009) ha encontrado que el nivel académico de los estudiantes de secundaria tiene efectos significativos sobre su estilo de aprendizaje. Finalmente, con respecto a la variable motivación, citar los trabajos de Martín-Díaz y Kempa (1991), donde se indica que los estudiantes prefieren diferentes estrategias de enseñanza de las ciencias en función de sus caracteristicas motivacionales, y de Méndez-Coca (2015), que pone de manifiesto que un enfoque de enseñanza centrado en el estudiante resulta más motivador que una metodología tradicional.

Acabamos de ver que, aunque no puede asegurarse que enseñar a los estudiantes en consonancia con sus estilos de aprendizaje mejore la eficacia instruccional, parece que todo apunta hacia la necesidad de conocer cómo quiere el estudiante de educación secundaria que se le enseñen las ciencias si se quiere conseguir, como mínimo, la motivación suficiente que permita su implicación en los procesos de aprendizaje. Dada la escasez de estudios sobre esta cuestión en los países iberoamericanos, nuestro propósito es analizar las preferencias instruccionales en la enseñanza de las ciencias de los estudiantes de educación secundaria. En concreto, nuestras preguntas de investigación son:

1. ¿Los estudiantes prefieren una enseñanza centrada en el profesor o una centrada en el estudiante?

2.¿Qué efectos tiene el nivel académico (la edad) del estudiante sobre la predileccion entre uno $u$ otro enfoque de enseñanza?

3. ¿Influye el sexo del estudiante en las preferencias de metodología de enseñanza?

Dados los claros indicios que señalan hacia un aumento de la motivación de los estudiantes cuando la enseñanza comporta su participacióna activa (metodologías centradas en el estudiante), es razonable pensar que los estudiantes ante opciones que ofrezcan la posibilidad de intervención activa del estudiante en los procesos de aprendizaje, escojan precisamente éstas y no otras que comporten pasividad y mera recepción de conocimientos. Por ello, nuestra primera hipótesis es:

H1: Los estudiantes preferirán metodologías de enseñanza centradas en el estudiante.

Atendiendo a que, como se ha dicho, en la educación secundaria la edad (nivel académico) lleva asociados cambios en las creencias epistemológicas y estilos de aprendizaje, la segunda hipótesis la podemos formular como:

H2: El nivel académico influirá de manera decisiva en las preferencias instruccionales, que cambiarán desde los primeros cursos de la educación secundaria hasta los últimos.

Por último, el género no ha evidenciado ser una variable significativa en cuanto a la predilección de actividades de aprendizaje o enfoques instruccionales, por esta razón nuestra tercera hipótesis es:

H3: El sexo no generará diferencias significativas en la elección de las metodologías de enseñanza de los estudiantes de secundaria.

\section{METODOLOGÍA}

\subsection{Diseño de la investigación}

Se trata de un estudio exploratorio con un diseño transversal de dos factores, esto es, hay dos variables independientes: el nivel académico (edad) y el género. La variable dependiente es la preferencia en la metodología de enseñanza.

\subsection{Sujetos participantes}

Han participado 240 estudiantes (112 mujeres y 128 hombres) de un centro educativo de educación secundaria de carácter privadoconcertado (privado pero subvencionado con fondos públicos), de los cuales 162 son de Educación Secundaria Obligatoria (ESO, período obligatorio de escolarización en España de cuatro cursos, que va desde los 12 a los 16 años; en el $4^{\circ}$ curso de la ESO los estudiantes pueden elegir entre un itinerario científico-tecnológico o uno humanístico-ciencias sociales), y 78 son de Bachillerato (período educativo no obligatorio en España en el que mayoritariamente los estudiantes se preparan para ir a la Universidad, 
es de dos cursos y va de los 16 a los 18 años; tiene cuatro modalidades: científico, científicotécnico, humanístico y ciencias sociales). Nuestro estudiantes son de $2^{\circ}, 3^{\circ}$ y $4^{\circ}$ de ESO (científico-tecnológico); y de $1^{\circ}$ y $2^{\circ}$ de Bachillerato (científico y científico-tecnológico).

El centro se halla ubicado en una ciudad grande (ciudad de Valencia), y el perfil socioeconómico de las familias del alumnado es medio-bajo. Estos sujetos no parecen tener, a priori, características especiales que los diferencien de estudiantes de otros centros. No obstante, hemos de indicar que no se realizó muestreo aleatorio alguno, ya que se trató de una muestra de conveniencia. Por ello, los resultados no pueden ser extrapolados a toda la población estudiantil, esto es, no hay garantías de validez externa.

\subsection{Materiales}

Se ha elaborado un cuestionario de opción múltiple compuesto por 6 ítems. Dichos ítems han sido extraídos del banco de ítems de Pedagogy of Science Inquiry Teaching Test de la Western Michigan University (Cobern et al., 2014), que está disponible en <http://www.wmich.edu/science/inquiryitems/>.

Los ítems están pensados para profesores, pero nosotros los hemos traducido al castellano y preparado para estudiantes. Cada ítem tiene cuatro opciones en las que para la situación problemática planteada se ofrece a los estudiantes cuatro posibilidades de enseñanza que van desde la totalmente centrada en el profesor hasta la totalmente centrada en el estudiante. Tiene dos opciones intermedias, una centrada en el profesor donde se da un cierto margen de participación activa al estudiante y otra, centrada en el estudiante, en la que el profesor dirige/orienta la enseñanza. De acuerdo con Pozo (1997), estas cuatro opciones podrían corresponder a la metodología de enseñanza tradicional (completamente centrada en el profesor), pasando por la metodología expositiva o de transmisión verbal de conocimientos (centrada en el profesor con alguna participación del estudiante) y la investigación dirigida (centrada en el estudiante con la implicación del profesor como guía), hasta la enseñanza por descubrimiento (completamente centrada en el estudiante). En los anexos pueden verse: a) las caracteríticas básicas de estos cuatro enfoques de enseñanza (Anexo 1); b) el cuestionario utilizado (Anexo 2); y c)la correspondencia entre cada una de las cuatro opciones de cada ítem con las distintas metodologías de enseñanza (Anexo 3).

\subsection{Procedimiento}

El cuestionario se administró durante una sesión de clase normal. Al principio de la clase se indicó a los estudiantes de que disponían de todo el tiempo necesario. También se informó previamente a los estudiantes de cómo se debía contestar el cuestionario, de la necesidad de leer con detenimiento cada una de las opciones y de ser sinceros en las respuestas. El tiempo que emplearon de media todos los cursos estuvo alrededor de los 30-35 minutos.

Con la finalidad de determinar la fiabilidad del cuestionario mediante el método de las dos mitades, tuvimos que evaluar cuantitativamente las respuestas de los ítems. Para ello, valoramos con un 1 punto la opción de la metodología tradicional, con 2 puntos la metodología expositiva, con 3 puntos la metodología de investigación dirigida y con 4 puntos la metodología de enseñanza por descubrimiento. Nótese que la puntuación es mayor cuanto más centrada está la metodología de enseñanza en el estudiante. Se tomaron los tres primeros ítems como primera mitad del cuestionario, y los tres últimos como la segunda mitad.

\section{RESULTADOS Y DISCUSIÓN}

En primer lugar, calculamos el coeficiente de correlación entre las dos mitades del cuestionario. Dicho coeficiente se calcula a partir de las puntuaciones obtenidas obtenidas por cada estudiante en cada una de las dos mitades, $y$ resultó ser $0.68 \quad$ (g.l. $=238, p<0.001)$. El coeficiente de fiabilidad de nuestro cuestionario, evaluado mediante la fórmula de SpearmanBrown, es de 0.81 , al que se le puede dar el calificativo de bueno.

En la Figura 1 se recogen los porcentajes (en forma acumulada) que se han obtenido en cada una de las metodologías de enseñanza a partir de las respuestas dadas por los estudiantes. Se ofrecen tanto los porcentajes por ítems como en el total del cuestionario.

Figura 1. Representación gráfica de los 


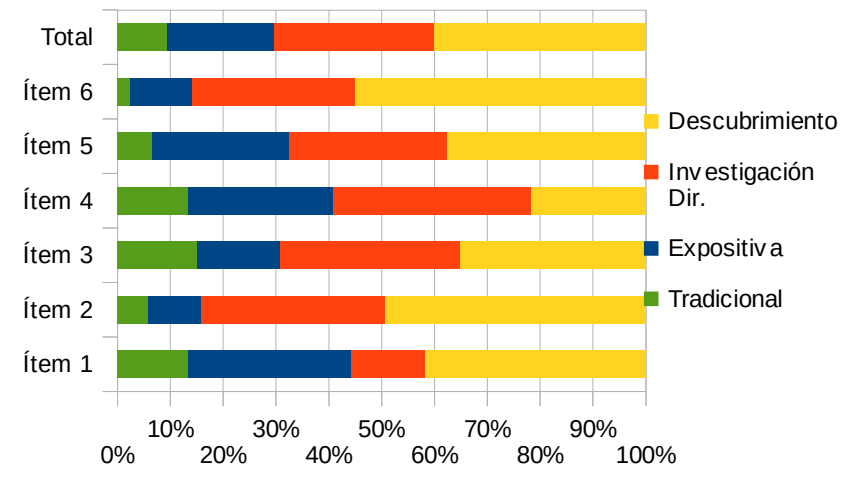

porcentajes de estudiantes, en forma acumulada, em cada metodología, en cada ítem y en el total del cuestionario.

Dadas las características de nuestro diseño de investigación y de nuestra variable dependiente, se ha usado la prueba estadística no paramétrica "chi cuadrado" a la hora de analizar la existencia de posibles relaciones entre variables o de diferencias significativas entre grupos. En concreto, se ha aplicado la prueba "chi cuadrado" a la frecuencia de sujetos que han elegido cada una de las metodologías de enseñanza en cada uno de los seis ítems. En todos los ítems han aparecido diferencias significativas entre metodologías de enseñanza, esto es, los estudiantes muestran preferencias significativas entre las distintas metodologías con $\mathrm{p}<0.001$ (ítem $1 \chi^{2}=54.27$, ítem $2 \chi^{2}=122.53$, ítem $3 \chi^{2}=35.33$, ítem $4 \chi^{2}=29.73$, ítem $5 \chi^{2}=$ 49.73 e ítem $6 \chi^{2}=155.33$, en todos los casos con g.l. $=3$ ).

Como puede observarse en la Figura 1, el origen de dichas diferencias significativas está en la predilección de los estudiantes por las metodologías centradas en el estudiante. De hecho, más de $70 \%$ del total de estudiantes prefieren una metodología centrada en el estudiante: $40 \%$ descubrmiento (D) y $30 \%$ investigación dirigida (ID). En tres de los seis ítems $(1,2$ y 6$)$ el porcentaje de $D$ es claramente mayor que el de ID. Solamente en uno, el ítem 4, la situación se invierte y predomina ID. En el resto, ítems 3 y 5 , están muy igualadas ambas metodologías. A destacar el bajo porcentaje de la metodología tradicional que no llega al $15 \%$ en ninguno de los ítems y el porcentaje relevante de sujetos, que casi llega al $30 \%$, que ha seleccionado la metodología expositiva $(E)$ en el ítem 4 y lo supera en el ítem 1 (este ítem es el único donde esta metodología $\mathrm{E}$ llega a superar a la ID). Estos resultados, que muestran una tendencia significativa de los estudiantes de secundaria hacia los enfoques de enseñanza centrados en el estudiante, están en sintonía con los estudios de McCombs y Whisler (1997), Daniels, Kalkman y McCombs (2001) y Alfassi (2004), que señalan un aumento de la motivación de los estudiantes ante estrategias instruccionales centradas en el estudiante.

En la Figura 2 aparecen representados los porcentajes acumulados en la metodología de enseñanza seleccionada por los estudiantes en función del nivel académico al que pertenecen.

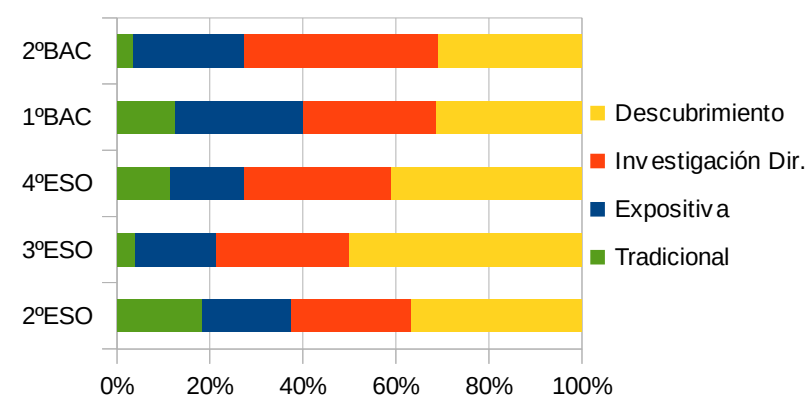

Figura 2. Representación gráfica de los porcentajes acumulados en la metodología seleccionada por los estudiantes en función del nivel académico al que pertenecen

Destacaremos algunos hechos relevantes de esta representación gráfica. En primer lugar, llama la atención que no se observa una tendencia definida hacia las centradas en el estudiante o el profesor con el aumento del nivel académico. No obstante, puede verse que la metodología por descubrimiento experimenta una reducción clara a partir de $1^{\circ}$ de Bachillerato (porcentajes en Bachillerato alrededor del 31\%, en tanto que en la ESO siempre son superiores al $37 \%$ llegando incluso al $50 \%$ en $3^{\circ}$ de ESO), al contrario de lo que ocurre con la metodología expositiva que aumenta a partir de dicho nivel (porcentajes en Bachillerato en torno al $25 \%$ y, en cambio sobre el $17 \%$ en la ESO). En las metodologías de investigación dirigida y tradicional no aparece un claro patrón de variación. Sin embargo, puede verse que a medida que se avanza en el nivel académico aumenta la predilección por las metodologías menos extremas, es decir por la expositiva y la investigación dirigida (la suma de los porcentajes de ambas va aumentando con el nivel 
académico, poco en la ESO y mucho en el Bachillerato ( $45 \%$ en $2^{\circ}$ de ESO; $46.15 \%$ en $3^{\circ}$ de ESO, $47.73 \%$ en $4^{\circ}$ de ESO; $56 \%$ en $1^{\circ}$ de Bachillerato y $65.48 \%$ en $2^{\circ}$ de Bachillerato).

La aplicación de la prueva "chi cuadrado" a la tabla de contingencia construida con el número de sujetos en cada nivel académico que escoge cada una de las cuatro metodologías nos da un valor de $\chi^{2}=89.99$, g.I. $=12, p<0.001$. Esto nos indica que la preferencia metodológica y el nivel académico están asociados de manera estadísticamente significativa. Dicho de otro modo, el nivel académico influye en la predilección de enfoque de enseñanza. Sin embargo, como ya hemos indicado, no hay una pauta definida de variación hacia las centradas en el estudiante o el profesor, aunque parece vislumbrarse que en los niveles académicos más altos se produce una reducción de la preferencia por la metodología por descubrimiento y un aumento de la expositiva. La relación de dependencia encontrada entre la preferencia de orientación metodológica y el nivel académico es coherente con los resultados de la investigación de Chen (2009). Dichos resultados ponen de manifiesto que el nivel académico es un factor que influye de manera significativa en las distintas dimensiones de los estilos de aprendizaje.

En la Figura 3 se ofrecen los porcentajes acumulados de estudiantes de ambos sexos que se decantan hacia cada una de las cuatro metodologías de enseñanza que figuran en el cuestionario.

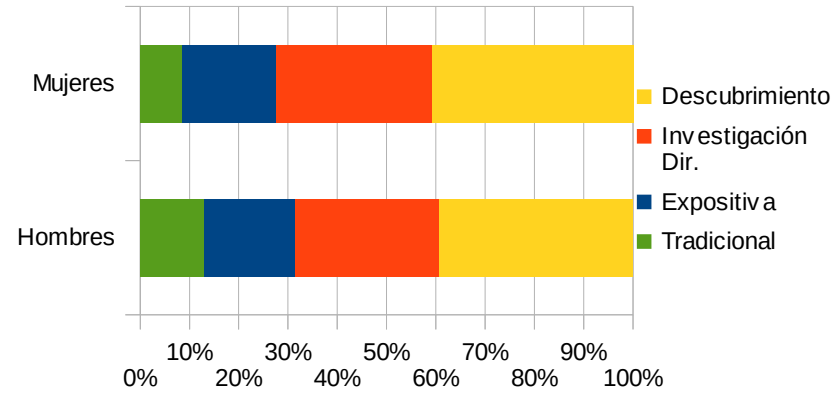

Figura 3. Representación gráfica de los porcentajes acumulados de estudiantes de ambos sexos que escogen cada una de las cuatro metodologías de enseñanza.

Como puede observarse las diferencias son mínimas entre hombre y mujeres. Si acaso comentar que parece que las mujeres muestran una mayor tendencia hacia los enfoques centrados en el estudiante $y$, sobre todo, les gusta menos la metodología tradicional. De hecho, la mayor diferencia de porcentajes, tanto en términos absolutos como en términos relativos, se da en la metodología tradicional.

La prueba chi cuadrado corrobora la independencia entre la variable género y la preferencia metodológica: $\chi^{2}=7.73$, g.I $=3, p=$ 0,052 . Es decir, se puede afirmar que el sexo no influye en la selección de la orientación de enseñanza. Este resultado está en completa consonancia con los estudios de Slater, Lujan y DiCarlo (2007), donde no aparecen diferencias significativas entre hombres y mujeres en las preferencias de estilos de aprendizaje; y de Weber y Custer (2005), que han encontrado una gran semejanza entre las preferencias de enfoques instruccionales de ambos sexos

\section{CONCLUSIONES E IMPLICACIONES DIDÁCTICAS}

En primer lugar, debemos señalar las límitaciones de este estudio exploratorio. La principal de estas limitaciones se deriva de la naturaleza y tamaño de la muestra (téngase en cuenta que se ha efectuado un muestreo de conveniencia $y$ ha participado solamente un centro educativo). Otra limitación puede tener su origen en el instrumento utilizado para conocer las preferencias en metodología de enseñanza de los estudiantes. Por todo ello, los resultados y las conclusiones de este estudio solamente pueden servir como primera aproximación.

De acuerdo con los porcentajes reflejados en la Figura 1 y con las pruebas estadísticas realizadas parece confirmarse nuestra primera hipótesis. Dicha hipótesis hacía referencia a que los estudiantes de educación secundaria mostrarían predilección por los enfoques instruccionales centrados en el estudiante. Efectivamente, dentro de los límites de nuestra investigación, todo indica que los estudiantes prefieren de forma estadísticamente significativa enfoques instruccionales centrados en el estudiante.

En cuanto a la influencia del nivel académico sobre la elección de metodología de enseñanza, los resultados recogidos en la Figura 2 y la prueba estadística "chi cuadrado" revelan una asociación significativa entre ambas 
variables. Nuestra segunda hipótesis apuntaba que la tendencia en la orientación metodológica cambiaría en función del nivel académico. En consecuencia, puede decirse que se verifica nuestra segunda hipótesis que, incluso, a tenor de nuestros resultados, podría matizarse en el sentido de que a medida que aumenta el nivel académico se incrementa la predilección por las metodologías menos extremas, esto es, aquellas que no están ni totalmente centradas en el estudiante ni totalmente centradas en el profesor.

La tercera hipótesis formulada estaba relacionada con la variable género. En concreto, señalaba que el sexo no produciría diferencias significativas en los modelos de instrucción preferidos por los estudiantes de secundaria. La prueba "chi cuadrado" realizada ha revelado la independencia de ambas variables y, por tanto, se puede concluir que la variable género no tiene efectos significativos sobre la selección del enfoque de enseñanza. Es decir, parece que nuestros resultados validan también nuestra tercera hipótesis. No obstante, no podemos dejar de indicar que las mujeres son menos partidarias que los hombres de la metodología tradicional.

Así pues los resultados obtenidos en este trabajo, aunque deben ser tomados con cierta prudencia dadas sus limitaciones, sacan a la luz de forma bastante clara la predilección de los estudiantes de secundaria por las metodologías centradas en el estudiante. Por otra parte, hay múltiples estudios que certifican que la utilización de estas metodologías aumenta tanto la motivación del estudiante (Lord, 1999; Meece, 2003; Mendez Coca, 2015; Tuan et al., 2005) como su rendimiento académico (Anjur, 2011; Armbruster et al., 2009; Granger et al., 2012; Michael, 2006). De ello se deduce que se ha de orientar al profesorado hacia dichas metodologías si se quiere atraer a los jóvenes hacia la ciencia y reducir los indices de fracaso en disciplinas científicas. Teniendo presente además el bajo nivel de alfabetización científica que muestran nuestros estudiantes al acabar la educación secundaria obligatoria (Solaz-Portolés y Selfa, 2015), resulta urgente sensibilizar a los docentes hacia la necesidad de cambiar sus estrategias de enseñanza en el aula. Sin embargo, no se puede obviar que el profesorado, incluso con formación didáctica de posgrado, se muestra reticente a introducir cambios en su modelos de instrucción centrados, en la mayoría de los casos, en el profesor
(Brines, Solaz-Portolés y Sanjosé, 2015; Solbes et al., 2013).

\section{REFERENCIAS}

1. Alfassi, M. Learning Environments Research, 2004, 7, 1.

2. Anjur, S.S. Advances in Physiology Education, 2011, 35, 161. http://advan.physiology.org/content/ajpadvan/3 5/2/161.full.pdf, consultada en enero 2015

3. Armbruster, P., Patel, M., Johnson, E., Weiss, M. CBE Life Sciences Education, 2009, 8 ,

203.

http://www.lifescied.org/content/8/3/203.full.pdf +html, consultada en febrero 2015

4. Bonwell, C. C., Eison, J. A. Active Learning: Creating Excitement in the Classroom, George Washington University Press: Washington, DC, 1991.

5. Brines, A., Solaz-Portolés, J. J., Sanjosé, V. Enseñanza de las Ciencias, 2016, 34, 107.

6. Brown, K. L. Education, 2003, 124, 49.

7. Bruffee, K. A. Collaborative learning: Higher education, interdependence, and the authority of knowledge, 2on Edition, Johns Hopkins University Press: Baltimore, 1999.

8. Cano, F. British Journal of Educational Psychology, 2005, 75, 203.

9. Catalano, G. D., Catalano, K. Journal of Engineering Education, 1999, 88, 59.

10. Chen, M.-L. Learning and Individual Differences, 2009, 19, 304.

11. Cobern, W. W., Schuster, D., Adams, B., Skjold, B. A., Muğaloğlu, E. Z., Bentz, A., Sparks, K. International Journal of Science Education, 2014, 36, 2265. .

12. Daniels, D.H.; Kalkman, D.L., McCombs, B.L. Early Education and Development, 2001, 12, 253.

13. Dunn, R., Dunn, K. Teaching secondary students through their individual learning styles: Practical approaches for grades 7-12. Allyn and Bacon: Boston, 1993.

14. Dunn, R., Griggs, S.A. Synthesis of the Dunn and Dunn learning-style model research: Who, what, when, where, and so what?, St.

PERIÓDICO TCHÊ QUÍMICA • www.periodico.tchequimica.com • Vol. 13 N. 26 - ISSN 1806-0374 (impresso) • ISSN 1806-9827 (CD-ROM) • ISSN 2179-0302 (meio eletrônico) 
John's University's Center for the Study of Learning and Teaching Styles: Jamaica, NY, 2007.

15. Felder, R.M., Henriques, E.R. Foreign Language Annals, 1995, 28, 21. http://www4.ncsu.edu/unity/lockers/users/f/feld er/public/Papers/FLAnnals.pdf, consultada en febrero 2015

16. Garcia-Ros, R., Pérez-González, F., Talaya-González, I. Revista Electrónica de Investigación Psicoeducativa, 2008, 16, 547. http://www.investigacion-

psicopedagogica.org/revista/new/ContadorArti culo.php?230, consultada en enero 2016

17. Gil, D. Enseñanza de las Ciencias, 1993, 11 ,

http://www.raco.cat/index.php/Ensenanza/articl e/view/21204/93254, consultada em abril 2015

18. Granger, E.M., Bevis, T.H.; Saka, Y., Southerland, S.A., Sampson, V., Tate, R. L. Science, 2012, 6103, 105.

19. Johnson, D.W., Johnson, R.T., Smith, K. A. Active Learning: Cooperation in the College Classroom, Interaction Book Company: Edina, MN, 1998.

20. Kempa, R. En Science Education: From Theory to Practice, Hofstein, A., Eylon, B.S., Giddings, G.J., eds., The Weizmann Institute of Science: Rehovot, Israel, 1995, pp. 119-123.

21. Lord, T.R. Journal of Environmental Education, 1999, 30, 22.

22. Martin-Diaz, M.J., Kempa, R. Enseñanza de las Ciencias, 1991, 9, 59. http://www.raco.cat/index.php/ensenanza/articl e/viewFile/51356/93105, consultada en marzo 2015

23. Marx, R.W., Blumenfeld, P.C., Krajcik, J. S., Fishman, B., Soloway, E., Geier, R., Tal, R.T. Journal of Research in Science Teaching, 2004, 41, 1063.

24. McCombs, B.L., Whisler, J.-S. The Learner-Centered Classroom and School: Strategies for Increasing Student Motivation and Achievement, Jossey-Bass Publishers: San Francisco, 1997.

25. Meece, J.L. Theory into Practice, 2003, 42, 109.
26. Méndez-Coca, D. Educación XX1, 2015, 18 , 215. http://revistas.uned.es/index.php/educacionXX 1/article/viewFile/14602/13002, consultada en abril 2015.

27. Michael, J. Advances in Physiology Education, 2006, 30, 159. http://advan.physiology.org/content/30/4/159.fu ll-text.pdf+html, consultada en abril de 2015.

28. Pashler, H., McDaniel, M., Rohrer, D., Bjork, R. Psychological Science in the Public Interest, 2009, 9, 105.

29. Porlán-Ariza, R., Rivero-García, A., Martín del Pozo, R. Enseñanza de las Ciencias, 1998, 16 ,

271. https://ddd.uab.cat/pub/edlc/02124521v16n2/0 2124521v16n2p271.pdf, consultada en enero 2015.

30. Pozo, J.I. Teorías cognitivas del aprendizaje, Morata: Madrid, 1997.

31. Rogowsky, B.A., Calhoun B.M., Tallal, P. Journal of Educational Psychology, 2015, 107, 64.

32. Slater, J.A., Lujan, H.L., DiCarlo, S.E. Advances in Physiology Education, 2007, 31, 336.

http://advan.physiology.org/content/ajpadvan/3 1/4/336.full.pdf, consultada en febrero 2015.

33. Solaz-Portolés, J.J., Selfa, B. (2016). Aceptado para su publicación en Revista de Pedagogía, 2016, 100.

34. Solbes, J., Domínguez-Sales, M.C., Fernández-Sánchez, J., Furió, C., Cantó, J.R., Guisasola, J. Didáctica de las Ciencias experimentales y Sociales, 2013, 27, 155. https://ojs.uv.es/index.php/dces/article/viewFile 12617/3317, consultada en enero 2015.

35. Tuan, H.-L., Chin C.-C., Tsai, C.-C., Cheng, S.-P. International Journal of Science and Mathematics Education, 2005, 3, 541.

36. Weber, K., Custer, R. Journal of Technology Education, 2005, 16, 55.

37. Weimar, M. Learner-Centered Teaching: Five Key Changes to Practice, 2nd ed., Jossey-Bass Publishers: San Francisco, 2013. 
Anexo 1. Algunos rasgos que caracterizan las cuatro enfoques de enseñanza a los que se hace referencia en este estudio

\begin{tabular}{|c|c|c|}
\hline \multirow[t]{3}{*}{ Tradicional } & $\begin{array}{l}\text { Actividades de } \\
\text { enseñanza }\end{array}$ & Basadas en la transmisión verbal \\
\hline & Papel del profesor & Proporciona conocimientos verbales \\
\hline & Papel del alumno & Recibe conocimientos y los reproduce \\
\hline \multirow[t]{3}{*}{ Expositiva } & $\begin{array}{l}\text { Actividades de } \\
\text { enseñanza }\end{array}$ & $\begin{array}{l}\text { Se centran en establecer relaciones entre } \\
\text { los conocimientos previos y los nuevos }\end{array}$ \\
\hline & Papel del profesor & $\begin{array}{l}\text { Proporciona los materiales y la } \\
\text { información necesaria }\end{array}$ \\
\hline & Papel del alumno & $\begin{array}{l}\text { Recibe los conocimientos de forma activa } \\
\text { y los estructura para asimilarlos }\end{array}$ \\
\hline \multirow[t]{3}{*}{$\begin{array}{l}\text { Investigación } \\
\text { orientada }\end{array}$} & $\begin{array}{l}\text { Actividades de } \\
\text { enseñanza }\end{array}$ & $\begin{array}{l}\text { Enseñanza mediante resolución guiada } \\
\text { de problemas }\end{array}$ \\
\hline & Papel del profesor & Plantea los problemas y dirige su solución \\
\hline & Papel del alumno & $\begin{array}{l}\text { Construye su conocimiento mediante la } \\
\text { investigación }\end{array}$ \\
\hline \multirow[t]{3}{*}{ Descubrimiento } & $\begin{array}{l}\text { Actividades de } \\
\text { enseñanza }\end{array}$ & $\begin{array}{l}\text { Basadas en la investigación y el } \\
\text { descubrimiento }\end{array}$ \\
\hline & Papel del profesor & Dirige la investigación \\
\hline & Papel del alumno & Investiga y busca sus propias respuestas \\
\hline
\end{tabular}

Anexo 2. Cuestionario utilizado en este estudio

Por favor, rellena este cuestionario marcando con una $\mathbf{X}$ la casilla que coincida con tu opinión.

\begin{tabular}{|l|l|}
\hline Curso & \\
\hline Grupo & \\
\hline
\end{tabular}

\begin{tabular}{|l|l|}
\hline Alumna \\
\hline Alumno \\
\hline
\end{tabular}




\section{Ítem 1. Fuerza y movimiento}

El profesor o la profesora va a introducir la relación entre fuerza y tipo de movimiento. De acuerdo con la segunda ley de Newton, una fuerza neta causará que un objeto acelere o se frene. Además se dispone de un carrito con carga al que se puede aplicar una fuerza.

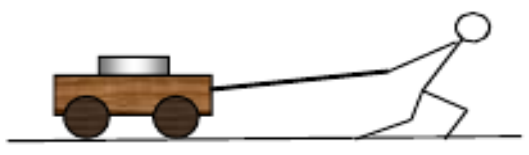

¿Cómo te gustaría que el profesor o la profesora tratara esta cuestión?

a.Preguntando qué movimiento ocurriría al aplicar una fuerza al carrito, y después los estudiantes lo empujarían o arrastrarían para comprobar qué ocurriría y proponer ellos mismos una ley física.

b.Escribiendo en la pizarra la segunda ley de Newton y explicándola de forma clara, y posteriormente los estudiantes podrían comprobar el tipo de movimiento empujando o arrastrando el carrito ellos mismos.

c.Preguntando si existe alguna relación entre fuerza y tipo de movimiento, y a continuación los estudiantes harían pruebas con el carrito y comentarían sus descubrimientos.

d.Escribiendo la segunda ley de Newton en la pizarra, explicándola claramente y demostrándolo empujando el carrito con una fuerza constante mientras observan los estudiantes.

\section{Ítem 2. Termómetros y su funcionamiento}

El profesor o la profesora quiere enseñar cómo funcionan los termómetros. En el laboratorio hay disponibles termómetros de verdad y también materiales para que los estudiantes construyan el suyo propio: una botellita, un tubito, un tapón agujereado y agua coloreada, además de agua caliente y agua fría.
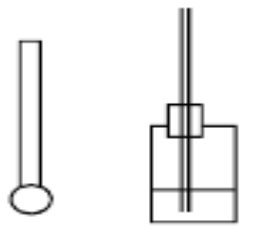

¿Cómo te gustaría que el profesor o la profesora tratara esta cuestión?

a.Dando el material a cada grupo de estudiantes, mostrando cómo se une cada pieza para que ellos mismos lo montasen. Una vez terminado explicarían su diseño al resto de la clase y su funcionamiento.

b.Mostrando a los estudiantes un termómetro ya montado y su funcionamiento, después entregándoles el material para que lo montaran ellos y lo comentasen.

c.Preguntando qué saben los estudiantes de los termómetros, dibujando uno y explicando cómo funciona, y después los estudiantes usarían sus termómetros.

d.Escribiendo en la pizarra el título "Termómetros" y dibujando el diagrama de uno de ellos, explicando cómo funcionan y contestando las preguntas de los estudiantes. Posteriormente se les enseñaría cómo funciona el termómetro de verdad. 


\section{Ítem 3. Disección de una rana}

El profesor o la profesora va a iniciar el tema de anatomía y propone la disección de una rana.

¿Cómo te gustaría que el profesor o la profesora tratara esta cuestión?

a.Permitiría a los estudiantes realizar la disección por ellos mismos, paso a paso, para explorar la anatomía de la rana y para que planteasen preguntas sobre el tema.

b.Explicando exactamente lo que los estudiantes necesitan saber acerca de la anatomía de la rana para que ellos mismos realizaran la disección.

c.Permitiría a los estudiantes realizar la disección, paso a paso, mientras contestan preguntas planteadas por el profesor o la profesora que ayudaría con comentarios y aclaraciones.

d.Llevaría a cabo una demostración de la disección, paso a paso, mientras indica claramente lo que hay que saber de la anatomía de la rana.

\section{Ítem 4. El aire es materia}

Los estudiantes han aprendido acerca de la materia. El profesor o la profesora va a enseñar que los gases (como lo es el aire) también son materia.

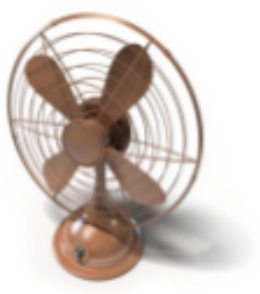

¿Cómo te gustaría que el profesor o la profesora tratara esta cuestión?

a.Preguntaría a los estudiantes si conocen alguna manera de comprobar si el aire es materia, utilizando equipo que haya en el aula o en el laboratorio, para que ellos prueben sus ideas.

b.Ayudaría a los estudiantes a encontrar formas de comprobar si el aire es materia, dejando que ellos hagan pruebas con ventiladores, sacando conclusiones de sus descubrimientos.

c.Comentaría a los estudiantes que el aire es materia y aunque no sea visible, se puede notar. A continuación los estudiantes utilizarían los ventiladores para comprobar si es cierto.

d.Explicaría a los estudiantes que el aire es materia y aunque no sea visible, se puede notar. A continuación el profesor o la profesora utilizaría los ventiladores para demostrarlo. 


\section{Ítem 5. Reflexión de la luz}

El profesor o la profesora va a enseñar la ley de la reflexión: "cuando un rayo de luz incide (choca) contra la superficie de un espejo, sale con un ángulo igual al que ha entrado". Para ello, se dispone de los siguientes materiales: un rayo láser, un espejo y un transportador de ángulos.

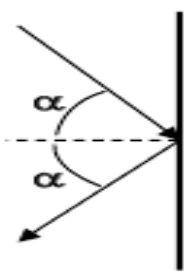

¿Cómo te gustaría que el profesor o la profesora tratara esta cuestión?

a.Escribiría la ley de la reflexión en la pizarra junto a un diagrama. Utilizaría los materiales disponibles y resolvería las dudas de los estudiantes.

b.Pediría a los estudiantes que hicieran ellos mismos el experimento con los materiales disponibles. Después los estudiantes comentarían lo que hubiesen descubierto.

c.Haría una pregunta sobre la reflexión y les daría los materiales disponibles. A continuación, los estudiantes comentarían lo que hubieran descubierto. Al final del tema les daría a los estudiantes un resumen sobre la ley de la reflexión.

d.Escribiría la ley de la reflexión en la pizarra junto a un diagrama. A continuación los estudiantes comprobarían la ley utilizando los materiales disponibles.

\section{Ítem 6. Volumen}

El profesor o la profesora quiere enseñar el concepto de volumen. Para este fin, desea comenzar el tema mostrando a sus estudiantes dos tarros de diferente tamaño (cada uno lleno de gominolas) y explicando que éstas pueden servir como instrumento para medir volúmenes simplemente contando las que caben.

\section{¿Cómo te gustaría que el profesor o la profesora tratara esta cuestión?}

a.Informaría a los alumnos del número exacto de gominolas que hay en cada tarro para que no haya errores.

b.Preguntaría cuál es el tarro que podría contener más gominolas y cómo harían para saberlo, después explicaría que las gominolas pueden ser un modo de medir el volumen.

c.Permitiría que los estudiantes rellenaran tarros de diferentes formas y tamaños, para luego exponer sus ideas sobre qué significa que haya diferente cantidad de gominolas en los tarros.

d.Explicaría que las gominolas pueden servir como instrumento de medida de volumen, ya que se puede comparar el volumen de cada tarro contando el número de gominolas. 
Anexo 3. Correspondencia entre cada una de las cuatro opciones de cada ítem con las distintas metodologías de enseñanza

\begin{tabular}{|c|c|c|c|c|}
\hline Ítem & A & B & C & D \\
\hline 1 & $\begin{array}{c}\text { Investigación } \\
\text { orientada }\end{array}$ & Expositiva & Descubrimiento & Tradicional \\
\hline 2 & Descubrimiento & $\begin{array}{c}\text { Investigación } \\
\text { orientada }\end{array}$ & Expositiva & Tradicional \\
\hline 3 & Descubrimiento & Expositiva & $\begin{array}{c}\text { Investigación } \\
\text { orientada }\end{array}$ & Tradicional \\
\hline 4 & Descubrimiento & $\begin{array}{c}\text { Investigación } \\
\text { orientada }\end{array}$ & Expositiva & Tradicional \\
\hline 5 & Tradicional & $\begin{array}{c}\text { Descubrimiento } \\
\text { Investigación } \\
\text { orientada }\end{array}$ & $\begin{array}{c}\text { Investigación } \\
\text { orientada }\end{array}$ & Expositiva \\
\hline 6 & Tradicional & Expositiva & \\
\hline
\end{tabular}

PERIÓDICO TCHÊ QUÍMICA • www.periodico.tchequimica.com • Vol. 13 N. 26.

• ISSN 1806-0374 (impresso) • ISSN 1806-9827 (CD-ROM) • ISSN 2179-0302 (meio eletrônico)

(C) 2016. Porto Alegre, RS. Brasil

The Periódico Tchê Química (ISSN: 1806-0374; 2179-0302) is an open-access journal since 2004. Journal DOI: 10.52571/PTQ. http://www.tchequimica.com. This text was introduced in this file in 2021 for compliance reasons.

○) The Author(s)
OPEN ACCESS. This article is licensed under a Creative Commons Attribution 4.0 (CC BY 4.0) International License, which permits use, sharing, adaptation, distribution, and reproduction in any medium or format, as long as you give approprate credi to the original auho(s) and Choure or exceeds the permitted use, you will need to obtain permission directly from the copyright holder. To view a copy of this license, visit http://creativecommons org/licenses/by/4.0/. 